\title{
Mental Load and Fatigue Assessment Instruments: A Systematic Review
}

\author{
Jesús Díaz-García ${ }^{1}{ }^{(0)}$, Inmaculada González-Ponce ${ }^{2, *}$, José Carlos Ponce-Bordón ${ }^{1}{ }^{(0)}$, \\ Miguel Ángel López-Gajardo ${ }^{1}$ (으, Iván Ramírez-Bravo ${ }^{1}$, Ana Rubio-Morales ${ }^{1}$ and Tomás García-Calvo ${ }^{1}$ (D)
}

Citation: Díaz-García, J.;

González-Ponce, I.; Ponce-Bordón, J.C.;

López-Gajardo, M.Á.

Ramírez-Bravo, I.; Rubio-Morales, A.; García-Calvo, T. Mental Load and

Fatigue Assessment Instruments: A

Systematic Review. Int. J. Environ.

Res. Public Health 2022, 19, 419.

https://doi.org/10.3390/

ijerph19010419

Academic Editor: Paul B.

Tchounwou

Received: 19 October 2021

Accepted: 28 December 2021

Published: 31 December 2021

Publisher's Note: MDPI stays neutral with regard to jurisdictional claims in published maps and institutional affiliations.

Copyright: (C) 2021 by the authors. Licensee MDPI, Basel, Switzerland. This article is an open access article distributed under the terms and conditions of the Creative Commons Attribution (CC BY) license (https:// creativecommons.org/licenses/by/ $4.0 /)$.
1 Faculty of Sport Sciences, University of Extremadura, 10003 Cáceres, Spain; jdiaz@unex.es (J.D.-G.); jponcebo@gmail.com (J.C.P.-B.); malopezgajardo@unex.es (M.Á.L.-G.); ivannramirezb@gmail.com (I.R.-B.); anarubmor94@gmail.com (A.R.-M.); tgarciac@unex.es (T.G.-C.)

2 Faculty of Education, University of Extremadura, 06006 Badajoz, Spain

* Correspondence: ingopo@unex.es

\begin{abstract}
Mental load and fatigue are important causes of performance decreases and accidents in different activities. However, a robust systematic review, detailing the instruments used to quantify them, is currently lacking. The purpose of this study was to summarize and classify by derivations the validated instruments used to quantify mental load and fatigue. The most representative electronic databases in the scope of this review, PubMed, WOS, Scopus, SPORTDiscus, and PsycINFO (until September 2020) were searched for studies that included instruments to analyze mental load and fatigue. The quality of the selected studies was scored using a quality assessment checklist. A total of 40 papers were included. Most of the papers used subjective scales $(75 \%)$ to quantify mental load and fatigue, with a small presence of behavioral $(n=5)$ and objective techniques $(n=5)$. Less is known about the analysis of mental load and fatigue using a combination of derivations. Despite the high cost and complexity of objective techniques, research that applies these measures is important for further analysis of brain processes in mental load and fatigue. The design of a battery of tests that include the three types of derivations also seems necessary.
\end{abstract}

Keywords: cognitive fatigue; mental health; assessment procedure; measurement; validity

\section{Introduction}

Fatigue can be caused by excessive mental and/or physical demands, but the analysis of fatigue has focused on physical aspects [1]. Physical fatigue causes impairments in the traditional physiological variables (i.e., heartrate, blood lactate, or oxygen uptake). Contrary to physical fatigue, mental fatigue is not associated with these impairments, although the specific role of the brain has been demonstrated in mental fatigue [2]. Mental fatigue is apparently caused by excessive mental demands [3], and some authors have used the term "mental load" to refer to it [4]. Mental load and fatigue have been widely associated with specific performance decreases or an increase in the risk of accidents [1,5]. Although the impairments caused by mental aspects reveal the importance of quantifying these variables, a robust systematic review of the available instruments used to quantify mental load and fatigue is currently lacking. This information may enhance the importance of controlling these variables and facilitate experts' choices of the most adequate instrument according to their needs.

Mental fatigue represents a psychobiological state with subjective (e.g., an increase in feelings of tiredness), behavioral (e.g., motivation decrease or reaction time increase), and physiological (e.g., alterations in the electroencephalogram signal) derivations in humans [6]. This psychobiological state is caused by brain-demanding tasks (i.e., mental load), with a relevant role of emotional (e.g., anxiety or stress) and cognitive (e.g., working memory or cognitive flexibility) aspects [6]. This should be considered in the analysis of mental load and fatigue, although most of the studies performed have used the cognitive 
aspects of mental fatigue [6]. For example, the case of Simon Biles or the different episodes observed during the COVID-19 are examples of how emotional aspects may impair health and performance.

Specifically, it has been observed that mental load and fatigue may impair human physical performance [6]. Some authors have stated that this phenomenon occurs through the increase in the subjective Ratio of Perceived Exertion, whereas other performance indicators, such as accuracy, tactical decisions, or reaction time, may be impaired by excessive accumulation of mental fatigue [1]. These impairments have been observed in different contexts such as medical surgery, construction work, or athletic settings [1]. Although the accumulation of extracellular adenosine or impairments in cognitive functions are possible explanations of this phenomenon, more studies are necessary to clarify the causes underlying these impairments.

However, it is difficult to analyze the causes and consequences of mental fatigue. Several covariables influence the mental fatigue induced by task performance, such as task difficulty, engagement, duration, or enjoyment/aversion [7]. In addition, a large number of individual differences could explain why the same task does not induce the same level of mental fatigue in different subjects or why mental fatigue manifests with different derivations (i.e., subjective, behavioral, or physiological) among participants [8]. Van Cutsem and Marcora [7] strongly recommend the use of a combination of several derivations (i.e., subjective, behavioral, and physiological) of mental fatigue as the best approach to identify its presence. Changes in all three areas do not necessarily appear in mentally fatiguing conditions, and they could depend on the subjects' individual characteristics. For example, cognitive performance does not necessarily decline in presence of mental fatigue due to the effect of the compensatory effort system [6-9]. Therefore, the use of different measures of mental fatigue may identify the causes of mental fatigue or explain why mental fatigue impairs performance.

Despite these recommendations, few existing procedures allow experts to assess mental load and fatigue, making them difficult to control [3]. On the one hand, different instruments have been used for this purpose indirectly, subjectively, and behaviorally. For mental load, we find the (i) NASA Task Load Index [10], (ii) the Subjective Mental Workload Scale (SCAM) developed by Ceballos-Vásquez et al. [11], or (iii) the "StuMMBE-Q" [12], among others. For mental fatigue, the subjectively reported Visual Analogue Scale (VAS) has been the most used instrument. Despite the high reliability and validity of these instruments, information about brain processes is lacking. On the other hand, objective instruments have also been used for this purpose. Pupil dilation [13], eye tracking [10], and different electrophysiological indicators such as electroencephalography (EEG; [14]) or brain functional connectivity patterns [15] have been recommended by authors to quantify mental load and fatigue. Although these measures are necessary to increase the quality of the data of mental fatigue, the high cost and the low ecological validity of the data extracted (e.g., EEG requires a sedentary activity to perform the measures; therefore, in a sport-specific context, experts cannot quantify the mental activity) of many of these instruments makes their use difficult.

\section{The Present Study}

Consequently, although the importance of mental variables in daily activities, work, or sports has increased because of the negative consequences of mental load and fatigue, it is difficult to choose a valid instrument to assess mental load and fatigue considering the different derivations caused by mental fatigue. Therefore, the first research question of this study is: What instruments exist to quantify mental load and mental fatigue? Consequently, the main purpose of this study was to summarize the instruments used to quantify mental load and fatigue. The second research question is: What instruments are more adequate to quantify each specific derivation of mental fatigue? Hence, we have also classified them by the type of derivation quantified to allow experts to choose the most adequate 
single instrument or battery of instruments, following the recommendations of using a combination of instruments for different derivations.

\section{Materials and Methods}

With this systematic review, we methodologically and comprehensively searched, appraised, and synthesized research evidence [16] for studies, aiming to identify the instruments used to quantify mental load and fatigue. This research was developed following the Preferred Report Elements for systematic reviews and meta-analyses (PRISMA) recommendations for systematic reviews [17] and the elements chosen for review [18]. Furthermore, this review was preregistered using the international prospective register of Systematic Reviews and Meta-analysis (PROSPERO [19,20]; registration: CRD42020167775).

\subsection{Eligibility Criteria and Search Strategy}

We followed the systematic review procedure suggested by Grant and Booth [16]. We included original empirical research papers published each year until September 2020 (i.e., we did not specify the start of the year, including all articles published until September 2020). Papers selected for analysis were found through searches of the most representative electronic databases for the scope of this review: PsycINFO, PubMed, Scopus, SPORTDiscus, and Web of Science. To identify the studies that used instruments to analyze mental load and mental fatigue, the authors used broad inclusion criteria, and all relevant research was included in the present study [21]. The following search terms were used to explore electronic journals: (i) mental load or mental fatigue; (ii) assessment OR measurement OR instrument; and (iii) validation. In each of the databases, the advanced search option was used to obtain the best combination and to access all possible research within our study framework. For example, in the Web of Science database, the following search was performed: TS = ("mental load" OR "mental fatigue") AND TS = ("assessment" OR "measurement" OR "instrument") AND TS = ("validation"). In addition to the search carried out in the databases, we performed a manual search to identify additional works to include in the study.

Before beginning the investigation, the inclusion and exclusion criteria were established to correctly define the objectives of this systematic review. Considering the search terms, we decided to include all the available works in each database, including all the languages present in each investigation. In addition, all articles published at any time before September 2020 were included. Another inclusion criterion was that all documents were original, with the full text available for analysis [22]. Articles with some measure or validity instrument on mental load or mental fatigue were also included.

\subsection{Study Selection and Data Collection Process}

Within the screening system, after reading the title and summary, the full text of the articles that were considered suitable for the review was selected to be evaluated and introduced into the study. The PRISMA flowchart (Figure 1) represents the filtering system for the final collection of the selected sample to complete the preparation of the current review. An expert meeting was held at each of the filters to determine the inclusion or exclusion of the different works previously analyzed. Figure 1 also shows the number of documents included and excluded in each of the phases of the screening process.

At the end of the search in the different databases, a total of 327 potential studies was obtained, of which 94 were eliminated for duplication and 158 due to the topic. Subsequently, 21 studies were eliminated for lack of the full text, and 14 because they did not meet the established quality criteria. After this selection process, a total of 40 articles was obtained.

All these steps were performed independently by two researchers following the same criteria. Kappa statistic $(k)$ was employed to test the percentage of interrater agreement, indicating strong agreement between the two raters $(k=0.85,[23,24])$. Discrepancies were discussed with a third reviewer until $100 \%$ consensus was reached. 


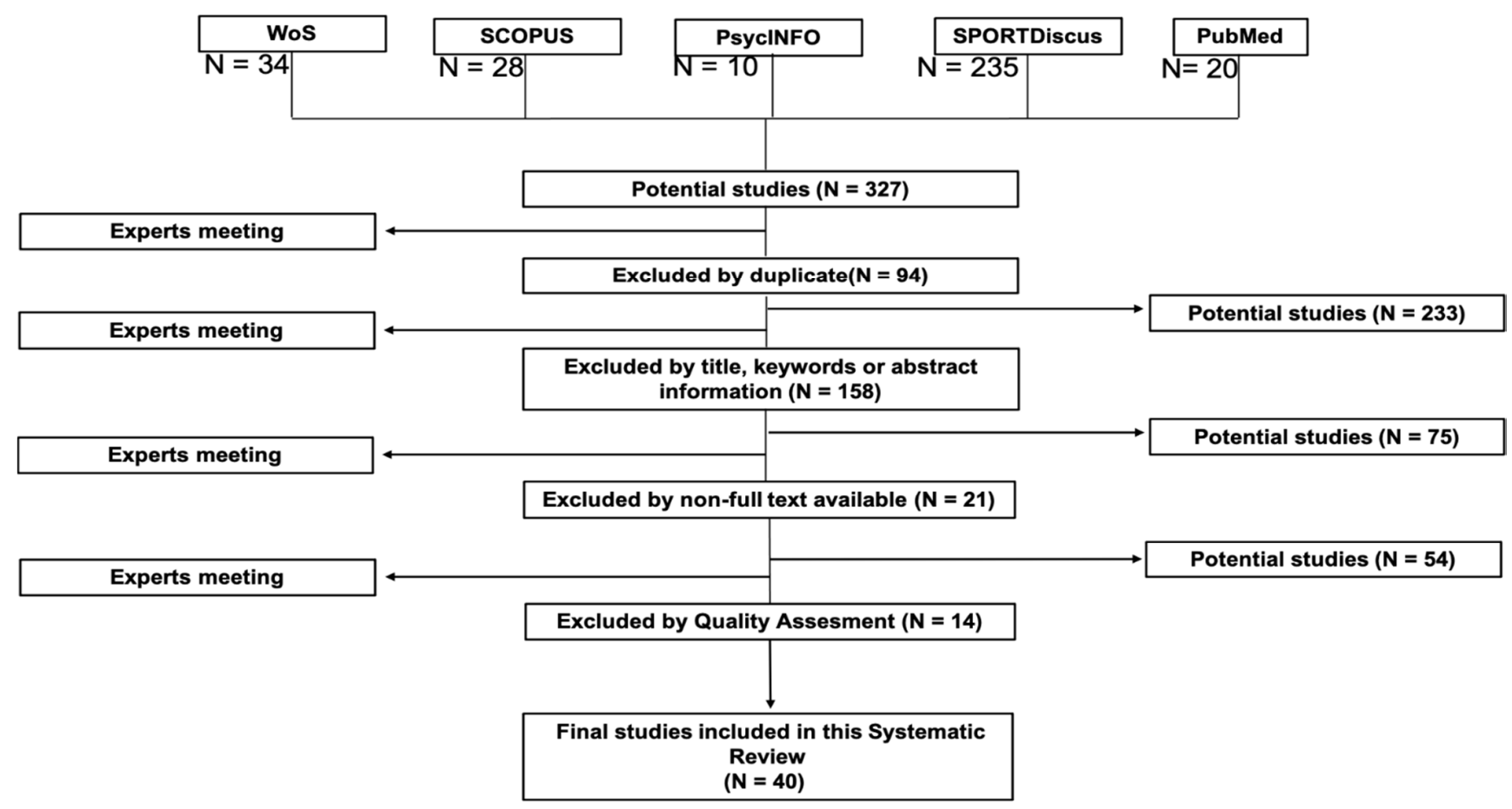

Figure 1. Process followed for the systematic review to classify by areas the validated instruments used to quantify mental load and fatigue.

\subsection{Data Synthesis}

Once the definitive studies were selected, a synthesis of the information and the most important characteristics of each article were extracted. Data relating to the instrument used, authors and year of publication, sample characteristics (i.e., number of volunteers, sex, age), and other instruments used for comparison were extracted. The results and conclusions to analyze the validity and reliability were collected. Thus, the studies were overviewed and compared, allowing us to evaluate the current state of research on mental load and mental fatigue assessment, which was divided into different sections. Due to the diversity of derivations through which mental load or mental fatigue was assessed, each document was assigned to one of the three subsequently established categories: (i) mental load and fatigue assessment instruments for subjective derivations; (ii) mental load and fatigue assessment instruments for behavioral derivations; (iii) mental load and fatigue assessment instruments for physiological derivations.

\subsection{Quality Assessment}

The quality of all studies was evaluated using the quantitative assessment tool 'QualSyst' [25]. This validated checklist consists of 14 sections, each assessing a different measurement property (see Table 1). Each item within a section is scored on a three-point scale depending on the degree to which the specific criteria were met (yes $=2$, partial $=1$, no $=0$ ). A score of $>0.75$ indicated strong quality, a score between 0.75 and 0.50 indicated moderate quality, and a score $<0.50$ indicated weak quality. The term "NA" was used for those items without a particular study design, which were excluded from the calculation of the summary score. This process was carried out by two reviewers (M.A.L.G. and J.C.P.B.), and discrepancies were discussed with a third reviewer (J.D.G.) until $100 \%$ consensus was reached. Likewise, the Kappa statistic $(\mathrm{k})$ was employed to test the percentage of interrater reliability [26]. These steps were performed by two reviewers. The agreement between researchers reflected in the kappa coefficient $(\kappa=0.84, k=0.85)$ indicated a strong initial agreement between the two raters $[23,24]$. Regarding quality assessments within individual studies, the kappa coefficient $(\kappa=0.91)$ indicated a strong initial agreement between the two raters $[23,24]$. Quality assess- 
ment of these 40 selected articles showed that 30 articles were of strong quality, 8 articles were of moderate quality, and 2 articles were of weak quality (see Table 1).

Table 1. Quality assessment 'Qualsyst'.

\begin{tabular}{|c|c|c|c|c|c|c|c|c|c|c|c|c|c|c|c|c|}
\hline Study & $\mathbf{A}$ & B & $\mathrm{C}$ & D & E & $\mathbf{F}$ & G & $\mathbf{H}$ & $\mathbf{I}$ & $\mathbf{J}$ & $\mathbf{K}$ & $\mathbf{L}$ & $\mathbf{M}$ & $\mathbf{N}$ & $\begin{array}{l}\text { Quality } \\
\text { Score }\end{array}$ & $\begin{array}{c}\text { Quality } \\
\text { Classification }\end{array}$ \\
\hline Chilcot et al. (2016) [27] & 2 & 1 & 2 & 1 & $\mathrm{~N} / \mathrm{A}$ & $\mathrm{N} / \mathrm{A}$ & $\mathrm{N} / \mathrm{A}$ & 2 & 1 & 2 & 2 & $\mathrm{~N} / \mathrm{A}$ & 2 & 2 & 0.77 & Strong \\
\hline Cho et al. (2007) [28] & 2 & 2 & 2 & 2 & $\mathrm{~N} / \mathrm{A}$ & $\mathrm{N} / \mathrm{A}$ & N/A & 2 & 2 & 1 & 1 & $\mathrm{~N} / \mathrm{A}$ & 2 & 1 & 0.77 & Strong \\
\hline Chiu et al. (2018) [29] & 2 & 2 & 2 & 2 & $\mathrm{~N} / \mathrm{A}$ & $\mathrm{N} / \mathrm{A}$ & $\mathrm{N} / \mathrm{A}$ & 2 & 1 & 2 & 1 & 2 & 2 & 2 & 0.91 & Strong \\
\hline Duan and $\mathrm{Mu}(2018)[30]$ & 2 & 2 & 2 & 2 & 0 & $\mathrm{~N} / \mathrm{A}$ & 0 & 2 & 2 & 2 & 2 & $\mathrm{~N} / \mathrm{A}$ & 2 & 2 & 0.83 & Strong \\
\hline Fong et al. (2015) [31] & 2 & 2 & 2 & 2 & 1 & $\mathrm{~N} / \mathrm{A}$ & $\mathrm{N} / \mathrm{A}$ & 2 & 2 & 2 & 2 & $\mathrm{~N} / \mathrm{A}$ & 2 & 2 & 0.95 & Strong \\
\hline Visser-Keizer et al. (2015) [32] & 2 & 2 & $\mathrm{~N} / \mathrm{A}$ & 2 & $\mathrm{~N} / \mathrm{A}$ & $\mathrm{N} / \mathrm{A}$ & $\mathrm{N} / \mathrm{A}$ & 2 & $\mathrm{~N} / \mathrm{A}$ & 2 & 2 & $\mathrm{~N} / \mathrm{A}$ & 2 & 2 & 1 & Strong \\
\hline Friedrich et al. (2018) [33] & 2 & 2 & 2 & 2 & $\mathrm{~N} / \mathrm{A}$ & $\mathrm{N} / \mathrm{A}$ & N/A & 2 & 2 & 2 & 2 & $\mathrm{~N} / \mathrm{A}$ & 2 & 2 & 1 & Strong \\
\hline Knobel et al. (2003) [34] & 2 & 2 & 2 & 2 & 2 & $\mathrm{~N} / \mathrm{A}$ & N/A & 2 & 2 & 2 & 2 & 2 & 2 & 2 & 1 & Strong \\
\hline Porro et al. (2019) [35] & 2 & 2 & 1 & 1 & $\mathrm{~N} / \mathrm{A}$ & $\mathrm{N} / \mathrm{A}$ & N/A & 2 & 1 & 2 & 2 & $\mathrm{~N} / \mathrm{A}$ & 2 & 2 & 0.85 & Strong \\
\hline Zhang et al. (2015) [36] & 2 & 2 & 1 & 2 & $\mathrm{~N} / \mathrm{A}$ & $\mathrm{N} / \mathrm{A}$ & 0 & 0 & 1 & 2 & $\mathrm{~N} / \mathrm{A}$ & $\mathrm{N} / \mathrm{A}$ & 1 & 2 & 0.65 & Moderate \\
\hline Kauffman et al. (2019) [37] & 2 & 2 & 2 & 2 & $\mathrm{~N} / \mathrm{A}$ & $\mathrm{N} / \mathrm{A}$ & $\mathrm{N} / \mathrm{A}$ & 2 & 2 & 2 & 2 & $\mathrm{~N} / \mathrm{A}$ & 2 & 2 & 1 & Strong \\
\hline Kumamoto and Arai (2004) [38] & 2 & 2 & 1 & 1 & $\mathrm{~N} / \mathrm{A}$ & $\mathrm{N} / \mathrm{A}$ & $\mathrm{N} / \mathrm{A}$ & 2 & 1 & 1 & 2 & $\mathrm{~N} / \mathrm{A}$ & 1 & 2 & 0.75 & Strong \\
\hline Dębska et al. (2013) [39] & 2 & 2 & 1 & 1 & $\mathrm{~N} / \mathrm{A}$ & 0 & 0 & 1 & 2 & 2 & 0 & $\mathrm{~N} / \mathrm{A}$ & 2 & 2 & 0.75 & Strong \\
\hline Bertram et al. (1990) [40] & 2 & 2 & 1 & 1 & $\mathrm{~N} / \mathrm{A}$ & $\mathrm{N} / \mathrm{A}$ & $\mathrm{N} / \mathrm{A}$ & 2 & 1 & 2 & 2 & $\mathrm{~N} / \mathrm{A}$ & 2 & 2 & 0.85 & Strong \\
\hline Chuang et al. (2018) [41] & 2 & 2 & 2 & 2 & $\mathrm{~N} / \mathrm{A}$ & $\mathrm{N} / \mathrm{A}$ & N/A & 2 & 2 & 2 & 2 & $\mathrm{~N} / \mathrm{A}$ & 2 & 2 & 1 & Strong \\
\hline Chung et al. (2014) [42] & 2 & 2 & 2 & 2 & 2 & $\mathrm{~N} / \mathrm{A}$ & $\mathrm{N} / \mathrm{A}$ & 2 & 2 & 2 & 2 & 1 & 2 & 2 & 0.96 & Strong \\
\hline Elbers et al. (2012) [43] & 2 & 2 & 2 & 2 & 2 & $\mathrm{~N} / \mathrm{A}$ & $\mathrm{N} / \mathrm{A}$ & 2 & 1 & 2 & 2 & 1 & 2 & 2 & 0.92 & Strong \\
\hline Hagelin et al. (2007) [44] & 2 & 2 & 2 & 1 & 1 & $\mathrm{~N} / \mathrm{A}$ & N/A & 2 & 2 & 2 & 2 & 1 & 2 & 1 & 0.83 & Strong \\
\hline Gentile et al. (2003) [45] & 2 & 2 & 2 & 2 & $\mathrm{~N} / \mathrm{A}$ & $\mathrm{N} / \mathrm{A}$ & $\mathrm{N} / \mathrm{A}$ & 1 & 2 & 2 & 2 & $\mathrm{~N} / \mathrm{A}$ & 2 & 2 & 1 & Strong \\
\hline Munch et al. (2006) [46] & 2 & 2 & 2 & 1 & $\mathrm{~N} / \mathrm{A}$ & $\mathrm{N} / \mathrm{A}$ & $\mathrm{N} / \mathrm{A}$ & 2 & 2 & 2 & 2 & $\mathrm{~N} / \mathrm{A}$ & 2 & 2 & 0.95 & Strong \\
\hline Schubart et al. (2019) [47] & 2 & 2 & 2 & 2 & $\mathrm{~N} / \mathrm{A}$ & $\mathrm{N} / \mathrm{A}$ & N/A & 2 & 2 & 2 & 2 & $\mathrm{~N} / \mathrm{A}$ & 2 & 2 & 1 & Strong \\
\hline Burke et al. (2018) [48] & 2 & 2 & 2 & 2 & $\mathrm{~N} / \mathrm{A}$ & $\mathrm{N} / \mathrm{A}$ & $\mathrm{N} / \mathrm{A}$ & 2 & 2 & 2 & 0 & $\mathrm{~N} / \mathrm{A}$ & 2 & 2 & 0.90 & Strong \\
\hline Krell (2017) [12] & $\mathrm{N} / \mathrm{A}$ & 2 & 2 & 2 & $\mathrm{~N} / \mathrm{A}$ & $\mathrm{N} / \mathrm{A}$ & $\mathrm{N} / \mathrm{A}$ & 2 & 2 & 2 & 2 & $\mathrm{~N} / \mathrm{A}$ & 2 & 2 & 0.90 & Strong \\
\hline Lin and Cai, (2009) [49] & 2 & 1 & $\mathrm{~N} / \mathrm{A}$ & $\mathrm{N} / \mathrm{A}$ & $\mathrm{N} / \mathrm{A}$ & $\mathrm{N} / \mathrm{A}$ & $\mathrm{N} / \mathrm{A}$ & $\mathrm{N} / \mathrm{A}$ & $\mathrm{N} / \mathrm{A}$ & 0 & $\mathrm{~N} / \mathrm{A}$ & $\mathrm{N} / \mathrm{A}$ & 0 & 0 & 0.30 & Weak \\
\hline Yang and $\mathrm{Wu}(2005)$ & 2 & 2 & 2 & 2 & N/A & $\mathrm{N} / \mathrm{A}$ & $\mathrm{N} / \mathrm{A}$ & 2 & 2 & 2 & $\mathrm{~N} / \mathrm{A}$ & $\mathrm{N} / \mathrm{A}$ & 2 & 2 & 0.90 & Strong \\
\hline Couvy-Duchesne et al. (2017) [51] & 2 & 2 & 2 & 1 & 2 & $\mathrm{~N} / \mathrm{A}$ & $\mathrm{N} / \mathrm{A}$ & 1 & 2 & 2 & 1 & 1 & 2 & 2 & 0.83 & Strong \\
\hline Shuman-Paretsky et al. (2017) [52] & 2 & 2 & 2 & 2 & $\mathrm{~N} / \mathrm{A}$ & $\mathrm{N} / \mathrm{A}$ & N/A & 2 & 2 & 2 & 2 & $\mathrm{~N} / \mathrm{A}$ & 2 & 2 & 1 & Strong \\
\hline Ceballos-Vásquez et al. (2016) [11] & 2 & 1 & 2 & 2 & $\mathrm{~N} / \mathrm{A}$ & $\mathrm{N} / \mathrm{A}$ & $\mathrm{N} / \mathrm{A}$ & 1 & 2 & 1 & 0 & $\mathrm{~N} / \mathrm{A}$ & 2 & 2 & 0.75 & Moderate \\
\hline MeAuley and Courneya (1994) [53] & 1 & 2 & 2 & 2 & $\mathrm{~N} / \mathrm{A}$ & $\mathrm{N} / \mathrm{A}$ & N/A & 1 & 2 & 1 & 2 & $\mathrm{~N} / \mathrm{A}$ & 2 & 2 & 0.85 & Strong \\
\hline Abma et al. (2013) [54] & 2 & 2 & 2 & 0 & $\mathrm{~N} / \mathrm{A}$ & 0 & N/A & 2 & 2 & 2 & $\mathrm{~N} / \mathrm{A}$ & $\mathrm{N} / \mathrm{A}$ & 2 & 2 & 0.80 & Strong \\
\hline Cimprich et al. (2011) [55] & 2 & 2 & 2 & 2 & $\mathrm{~N} / \mathrm{A}$ & $\mathrm{N} / \mathrm{A}$ & $\mathrm{N} / \mathrm{A}$ & 1 & 2 & 2 & 2 & $\mathrm{~N} / \mathrm{A}$ & 2 & 2 & 0.79 & Strong \\
\hline Di Stasi et al. (2012) [56] & 2 & 1 & 1 & 2 & 0 & 0 & 0 & 0 & 0 & 2 & 0 & $\mathrm{~N} / \mathrm{A}$ & 2 & 2 & 0.46 & Weak \\
\hline Puspasari et al. (2017) [57] & 2 & 2 & 1 & 1 & 0 & 0 & 0 & 2 & 1 & 2 & 1 & $\mathrm{~N} / \mathrm{A}$ & 2 & 2 & 0.62 & Moderate \\
\hline Price et al. (2017) [58] & 1 & 2 & 2 & 2 & 2 & 0 & 2 & 2 & 0 & 2 & 1 & $\mathrm{~N} / \mathrm{A}$ & 2 & 2 & 0.77 & Strong \\
\hline Crocetta et al. (2014) [59] & 2 & 1 & 2 & 2 & $\mathrm{~N} / \mathrm{A}$ & $\mathrm{N} / \mathrm{A}$ & $\mathrm{N} / \mathrm{A}$ & 2 & 2 & 2 & 2 & $\mathrm{~N} / \mathrm{A}$ & 2 & 2 & 0.95 & Strong \\
\hline Neal et al. (2014) [60] & 2 & 2 & 1 & 2 & 0 & 0 & 0 & 2 & 1 & 2 & 2 & $\mathrm{~N} / \mathrm{A}$ & 2 & 2 & 0.69 & Moderate \\
\hline Liu et al. (2016) [61] & 2 & 2 & $\mathrm{~N} / \mathrm{A}$ & 1 & $\mathrm{~N} / \mathrm{A}$ & $\mathrm{N} / \mathrm{A}$ & $\mathrm{N} / \mathrm{A}$ & $\mathrm{N} / \mathrm{A}$ & $\mathrm{N} / \mathrm{A}$ & 2 & $\mathrm{~N} / \mathrm{A}$ & $\mathrm{N} / \mathrm{A}$ & 2 & 2 & 0.69 & Moderate \\
\hline Gharagozlou et al. (2015) [62] & 2 & 2 & 2 & 1 & $\mathrm{~N} / \mathrm{A}$ & 0 & 0 & 0 & 1 & 2 & 0 & $\mathrm{~N} / \mathrm{A}$ & 2 & 2 & 0.58 & Moderate \\
\hline Patel et al. (2018) [25] & 2 & 2 & 1 & 1 & 0 & 0 & 0 & 1 & 1 & 2 & 1 & $\mathrm{~N} / \mathrm{A}$ & 1 & 2 & 0.54 & Moderate \\
\hline Sun et al. (2014) [15] & 1 & $\mathrm{~N} /$ & $\mathrm{AN} / \mathrm{A}$ & 2 & $\mathrm{~N} / \mathrm{A}$ & $\mathrm{N} / \mathrm{A}$ & $\mathrm{N} / \mathrm{A}$ & 2 & $\mathrm{~N} / \mathrm{A}$ & 2 & 2 & $\mathrm{~N} / \mathrm{A}$ & 2 & 2 & 0.59 & Moderate \\
\hline
\end{tabular}

Note. Articles were presented in the same order as the Tables' results. Criteria $=1$. Alphabetical order of the instrument; 2. Alphabetical order of the authors. Punctuations: Yes $=0$; partial $=1$, no $=0$. Variables: A $=$ Question described; $\mathrm{B}=$ Appropiate study design; $\mathrm{C}=$ Appropiate subject selection; $\mathrm{D}=$ Characteristics described; $\mathrm{E}=$ Random allocation; $\mathrm{F}=$ Researchers blinded; $\mathrm{G}=$ Subjects blinded; $\mathrm{H}=$ Outcome measures well defined and robust againts bias $\mathrm{I}$ = Sample size appropiate; $\mathrm{J}$ = Analytic methods well described; $\mathrm{K}=$ Estimate of variance reported; $\mathrm{L}=\mathrm{Controlled}$ for confounding variables; $\mathrm{M}=$ Results reported details; $\mathrm{N}=$ Conclusions reported by results.

\section{Results}

\subsection{Mental Load and Mental Fatigue Assessment Instruments for Subjective Derivations}

Table 2 shows the studies $(n=31)$ that have used and tested instruments for the subjective derivation of mental load and fatigue. Of these studies, $75 \%$ focused on subjective derivation. However, we observed that most of these instruments focused on terms related to mental load and fatigue but not on these specific terms. These related terms are, for example, job-related stress (e.g., Mental Workload Instrument or Fatigue Assessment Scale for Construction Workers), or chronic fatigue syndrome. Concerning the variables and instruments used to validate these instruments, most of these studies used other related scales such as the Ratio of Perceived Exertion to compare the results obtained. Some authors used the behavioral consequences of mental fatigue, such as sleep (i.e., PSQI), in comparison with the CFS and biological parameters (i.e., ECG). The population used to validate these instruments ranged from school and university students to workers and clinical patients. 
Table 2. Mental load and mental fatigue assessment instruments for subjective derivations.

\begin{tabular}{|c|c|c|c|c|c|c|}
\hline $\begin{array}{l}\text { Mental Load or } \\
\text { Fatigue Instrument }\end{array}$ & Authors & Sample & & $\begin{array}{l}\text { struments Used to } \\
\text { Compare }\end{array}$ & Results & Conclusions \\
\hline \multirow[t]{2}{*}{$\begin{array}{l}\text { Chalder Fatigue } \\
\text { Questionnaire (CFQ) }\end{array}$} & Chilcot et al. (2016) & $\begin{array}{l}444 \text { participants with } \\
\text { multiple sclerosis } \\
(M=45.15, S D=12.35)\end{array}$ & $\begin{array}{l}1 . \\
2 . \\
3 . \\
4 .\end{array}$ & $\begin{array}{l}\text { CFQ } \\
\text { Work and Social } \\
\text { Adjustment Scale } \\
\text { (WSAS) } \\
\text { Multidimensional } \\
\text { Fatigue Inventory } \\
\text { (MFI) } \\
\text { Hospital Anxiety and } \\
\text { Depression Scale } \\
\text { (HADS). }\end{array}$ & $\begin{array}{l}\text { Reliability coefficients for } \\
\text { mental and physical } \\
\text { subscales were both } 0.96 \text {. }\end{array}$ & $\begin{array}{l}\text { CFQ is a valid and reliable } \\
\text { instrument to measure } \\
\text { fatigue severity in people } \\
\text { with multiple sclerosis. }\end{array}$ \\
\hline & Cho et al. (2007) & $\begin{array}{l}207 \text { primary care } \\
\text { patients, between } 18 \\
\text { and } 45 \text { years old. }\end{array}$ & $\begin{array}{l}1 . \\
2 .\end{array}$ & $\begin{array}{l}\text { 12-item General } \\
\text { Health Questionnaire } \\
\text { (GHQ-12) } \\
\text { Revised Clinical } \\
\text { Interview Schedule } \\
\text { (CIS-R). }\end{array}$ & $\begin{array}{l}\text { The Brazilian CFQ's } \\
\text { internal consistency } \\
\text { improved slightly from } \\
\text { the pilot study to the } \\
\text { validation study: } \\
\text { Cronbach's alpha from } \\
0.86 \text { to } 0.88 \text {. }\end{array}$ & $\begin{array}{l}\text { Brazilian CFQ had good } \\
\text { reliability and validity, which } \\
\text { have improved during the } \\
\text { intercultural adaptation and } \\
\text { validation process. }\end{array}$ \\
\hline $\begin{array}{l}\text { Chinese Mental } \\
\text { Fatigue Scale (CMFS) }\end{array}$ & Chiu et al. (2018) & $\begin{array}{l}150 \text { traumatic } \\
\text { brain-injured adults } \\
\text { for } 6 \text { months } \\
(M=50.90) .\end{array}$ & 1. & $\begin{array}{l}\text { Clinical Useful } \\
\text { Depression Outcome } \\
\text { Scale Chinese version } \\
\text { (CUDOS) }\end{array}$ & $\begin{array}{l}\text { Correlations between the } \\
\text { items and the total scores } \\
\text { ranged from } 0.48 \text { to } 0.81 \\
\text { for the 13-item MFS (all } \\
p<0.001 \text { ). }\end{array}$ & $\begin{array}{l}\text { CMFS has satisfactory } \\
\text { statistical properties to } \\
\text { quantify mental fatigue in } \\
\text { traumatic brain-injured } \\
\text { patients. }\end{array}$ \\
\hline $\begin{array}{l}\text { Chinese version of } \\
\text { Stress Overload } \\
\text { Scale-Short(SOS-SC) }\end{array}$ & $\begin{array}{l}\text { Duan and } \mathrm{Mu} \\
\text { (2018) }\end{array}$ & $\begin{array}{l}1364 \text { adults } \\
(M=40.00 ; S D=7.60)\end{array}$ & $\begin{array}{l}1 . \\
2 . \\
3 . \\
4 .\end{array}$ & $\begin{array}{l}\text { Multidimen-sional } \\
\text { Scale of Perceived } \\
\text { Social Sup-port } \\
\text { Depression Anxiety } \\
\text { Stress Scale } \\
\text { Brief Inventory of } \\
\text { Thriving. }\end{array}$ & $\begin{array}{l}\text { Personal vulnerability and } \\
\text { workload were positively } \\
\text { and significantly } \\
\text { correlated with the score } \\
\text { of SOS-SC. }\end{array}$ & $\begin{array}{l}\text { SOS-SC can be used to } \\
\text { measure stress and mental } \\
\text { health status in the } \\
\text { Chinese population. }\end{array}$ \\
\hline \multirow[b]{2}{*}{$\begin{array}{l}\text { Chronic Fatigue } \\
\text { Syndrome (CFS) } \\
\text { Chinese Version }\end{array}$} & Chiu et al. (2018) & $\begin{array}{l}150 \text { traumatic } \\
\text { brain-injured adults } \\
\text { for } 6 \text { months } \\
(M=50.90) .\end{array}$ & 1. & CUDOS & $\begin{array}{l}\text { Correlations between the } \\
\text { items and the total scores } \\
\text { ranged from } 0.39 \text { to } 0.81 \\
\text { for the } 14 \text {-item CMFS } \\
(p<0.001)\end{array}$ & $\begin{array}{l}\text { CFS has satisfactory } \\
\text { statistical properties to } \\
\text { quantify mental fatigue in } \\
\text { traumatic brain-injured } \\
\text { patients. }\end{array}$ \\
\hline & Fong et al. (2015) & $\begin{array}{l}1259 \text { adults from } \\
\text { different jobs } \\
(M=43.0, S D=8.0)\end{array}$ & $\begin{array}{l}1 . \\
2 . \\
3 . \\
4 .\end{array}$ & $\begin{array}{l}\text { 4-point Chinese } \\
\text { Hospital Anxiety } \\
\text { Depression } \\
\text { Scale19-item Chinese } \\
\text { Pittsburgh Sleep } \\
\text { Quality Index (PSQI) } \\
\text { 12-item Chinese } \\
\text { Short-Form Health } \\
\text { Survey. }\end{array}$ & $\begin{array}{l}\text { Three factors of CFS } \\
\text { (physical fatigue, low } \\
\text { energy, and mental } \\
\text { fatigue) were positively } \\
\text { correlated with anxiety } \\
(r=0.32-0.47, p<0.01) \text {, } \\
\text { depression }(r=0.31-0.50, \\
p<0.01) \text {, and exhaustion } \\
(r=0.41-0.59, p<0.01) \text {, } \\
\text { and weakly correlated } \\
\text { with sleep disorders } \\
(r=0.21-0.30, p<0.01) .\end{array}$ & $\begin{array}{l}\text { CFS is a valid measure of } \\
\text { fatigue symptoms in the } \\
\text { general population. }\end{array}$ \\
\hline $\begin{array}{l}\text { Dutch Multifactor } \\
\text { Fatigue Scale }\end{array}$ & $\begin{array}{l}\text { Visser-Keizer et al. } \\
(2015)\end{array}$ & $\begin{array}{l}148 \text { participants, } 9 \\
\text { with stroke, } 5 \text { with } \\
\text { traumatic brain injury, } \\
55 \text { with ischemic } \\
\text { stroke, } 22 \text { with } \\
\text { hemorrhagic stroke, } \\
22 \text { with acquired } \\
\text { brain injury, and } 35 \\
\text { with traumatic brain } \\
\text { injury. }\end{array}$ & No & & $\begin{array}{l}\text { Good reliability is shown } \\
\text { for mental fatigue } \\
(\text { ICC }>0.80) \text {. Patients } \\
\text { without injuries reported } \\
\text { significantly greater } \\
\text { mental fatigue than } \\
\text { patients with injury. }\end{array}$ & $\begin{array}{l}\text { This questionnaire } \\
\text { diagnoses fatigue. }\end{array}$ \\
\hline
\end{tabular}


Table 2. Cont.

\begin{tabular}{|c|c|c|c|c|c|c|}
\hline $\begin{array}{c}\text { Mental Load or } \\
\text { Fatigue Instrument }\end{array}$ & Authors & Sample & & $\begin{array}{c}\text { Instruments Used to } \\
\text { Compare }\end{array}$ & Results & Conclusions \\
\hline \multirow{3}{*}{$\begin{array}{l}\text { EORTC QLQ-FA12 } \\
\text { quality of life } \\
\text { questionnaire }\end{array}$} & $\begin{array}{l}\text { Friedrich et al. } \\
\text { (2018) }\end{array}$ & $\begin{array}{l}577 \text { participants } \\
(M=30.3, S D=6.1)\end{array}$ & $\begin{array}{l}1 . \\
2 . \\
3 .\end{array}$ & $\begin{array}{l}\text { EORTC QLQ-C30 } \\
\text { questionnaire. } \\
\text { HADS. } \\
\text { Supportive Care } \\
\text { Needs Short Form } 34 \\
\text { items. }\end{array}$ & $\begin{array}{l}\text { The cognitive fatigue } \\
\text { items' reliability ranged } \\
\text { from } 0.45 \text { to } 0.73 \text {. The } \\
\text { correlations between the } \\
\text { three scales ranged } \\
\text { between } 0.63 \text { and } 0.70 \text {. } \\
\text { Cronbach's alpha for } \\
\text { cognitive fatigue was } 0.73 \text {. }\end{array}$ & $\begin{array}{l}\text { This instrument can } \\
\text { discriminate between } \\
\text { physical, emotional, and } \\
\text { cognitive fatigue. }\end{array}$ \\
\hline & Knobel et al. (2003) & $\begin{array}{l}238 \text { advanced cancer } \\
\text { patients and } 128 \\
\text { cancer survivors } \\
(M=52.50)\end{array}$ & 1. & $\begin{array}{l}\text { Fatigue Questionnaire } \\
\text { (FQ) }\end{array}$ & $\begin{array}{l}\text { FA scale correlated } \\
\text { between } 0.49 \text { and } 0.75 \text { at } \\
\text { all assessment points with } \\
\text { the Physical Fatigue (PF) } \\
\text { and Mental Fatigue (MF) } \\
\text { scales of the FQ. }\end{array}$ & $\begin{array}{l}\text { EORTC QLQ C } 30 \text { fatigue } \\
\text { scale meaasures fatigue as } \\
\text { part of an overall fatigue } \\
\text { assessment. }\end{array}$ \\
\hline & Porro et al. (2019) & $\begin{array}{l}68 \text { breast cancer } \\
\text { patients }(M=46.97 \\
S D=6.92)\end{array}$ & 1. & MFI-20. & $\begin{array}{l}\text { Univariate analyzes } \\
\text { showed Return To Work } \\
\text { (RTW) probability was } \\
\text { reduced by high scores for } \\
\text { mental fatigue, } r=0.85 \text {, } \\
p<0.05 \text {. Only the change } \\
\text { in mental fatigue during } \\
\text { treatment influenced the } \\
\text { RTW probability. }\end{array}$ & $\begin{array}{l}\text { Attention should be paid } \\
\text { to the use of validated } \\
\text { scales to evaluate mental } \\
\text { constructs. }\end{array}$ \\
\hline $\begin{array}{l}\text { Fatigue Assessment } \\
\text { Scale for Construction } \\
\text { Workers (FASCW). }\end{array}$ & Zhang et al. (2015) & $\begin{array}{l}144 \text { unionized } \\
\text { construction workers } \\
\text { in New England, } \\
\text { from } 19 \text { to } 60 \text { years } \\
(M=42.4 ; S D=10.3) .\end{array}$ & $\begin{array}{l}1 . \\
2 .\end{array}$ & $\begin{array}{l}\text { Ratio of Perceived } \\
\text { Exertion (RPE) } \\
\text { Profile of Mood States } \\
\text { (POMS). }\end{array}$ & $\begin{array}{l}\text { Results indicated } \\
\text { significant high } \\
\text { correlations between } \\
\text { FASCW and the Fatigue } \\
\text { subscale of POMS and the } \\
\text { measure of RPE. }\end{array}$ & $\begin{array}{l}\text { FASCW is a promising } \\
\text { instrument for assessing a } \\
\text { general concept of fatigue. }\end{array}$ \\
\hline $\begin{array}{l}\text { Functional Status } \\
\text { Questionnaire }\end{array}$ & $\begin{array}{l}\text { Kauffman et al. } \\
\text { (2019) }\end{array}$ & $\begin{array}{l}1287 \text { undergraduate } \\
\text { students }(M=21.68 \\
S D=4.54)\end{array}$ & $\begin{array}{l}1 . \\
2 . \\
3 .\end{array}$ & $\begin{array}{l}\text { Anxiety Sensitivity } \\
\text { Index } \\
\text { Inventory of } \\
\text { Depression and } \\
\text { Anxiety Symptoms } \\
\text { Positive and Negative } \\
\text { Affect Schedule } \\
\text { (PANAS). }\end{array}$ & $\begin{array}{l}\text { FSQ had excellent internal } \\
\text { consistency }(\alpha=0.92) . \\
\text { Total FSQ score was } \\
\text { positively associated with } \\
\text { anxiety sensitivity } \\
(r=0.49), \text { general } \\
\text { depression }(r=0.37), \\
\text { social anxiety }(r=0.40), \\
\text { panic }(r=0.43), \text { and } \\
\text { negative affectivity } \\
(r=0.37) .\end{array}$ & $\begin{array}{l}\text { FSQ may be a valid and } \\
\text { promising approach to } \\
\text { better understand the } \\
\text { implications of fatigue in } \\
\text { real-world contexts (e.g., } \\
\text { primary care). }\end{array}$ \\
\hline J-ZBI-8 Questionnaire & $\begin{array}{l}\text { Kumamoto and } \\
\text { Arai (2004) }\end{array}$ & $\begin{array}{l}315 \text { subjects who } \\
\text { lived with primary } \\
\text { caregivers }(M=81.2 \text {, } \\
S D=7.5)\end{array}$ & No & & $\begin{array}{l}\text { No clear relationship } \\
\text { between the nursing care } \\
\text { load and nursing time was } \\
\text { found. This relationship is } \\
\text { significantly related to the } \\
\text { attention load. }\end{array}$ & $\begin{array}{l}\text { The J-ZBI__ } \\
\text { questionnaire has two } \\
\text { subscales whose factorial } \\
\text { structure is clearly } \\
\text { defined. }\end{array}$ \\
\hline $\begin{array}{l}\text { Meister } \\
\text { Questionnaire }\end{array}$ & Dębska et al. (2013) & $\begin{array}{l}211 \text { nurses }(M=43.1 \\
S D=7.26)\end{array}$ & 1. & $\begin{array}{l}\text { Maslach Burnout } \\
\text { Inventory. }\end{array}$ & $\begin{array}{l}\text { Cronbach's alpha was } 0.83 \\
\text { for the total score. }\end{array}$ & $\begin{array}{l}\text { Meister questionnaire } \\
\text { meets the psychometric } \\
\text { criteria of reliability and } \\
\text { validity to assess mental } \\
\text { load in nurses. }\end{array}$ \\
\hline $\begin{array}{l}\text { Mental WorkLoad } \\
\text { Instrument. }\end{array}$ & $\begin{array}{l}\text { Bertram et al. } \\
\text { (1990) }\end{array}$ & $\begin{array}{l}48 \text { patients admitted } \\
\text { to clinical care, } \\
\text { between } 31 \text { and } 45 \\
\text { years old. }\end{array}$ & No & & $\begin{array}{l}\text { Significant correlations } \\
\text { were observed between } \\
\text { the work demand, } \\
\text { satisfaction, and } \\
\text { self-perceived } \\
\text { performance. }\end{array}$ & $\begin{array}{l}\text { Mental workload } \\
\text { correlated directly and } \\
\text { inversely with both } \\
\text { satisfaction and the } \\
\text { self-rated quality of the } \\
\text { patient care provided. }\end{array}$ \\
\hline MFI & $\begin{array}{l}\text { Chuang et al. } \\
\text { (2018) }\end{array}$ & $\begin{array}{l}123 \text { participants ( } 43 \\
\text { males and } 80 \text { females; } \\
M=46.12, S D=18.40 \text { ) }\end{array}$ & 1. & $\begin{array}{l}\text { PSQI } \\
\text { Survey of Short } \\
\text { Format Health } \\
\text { (SF-36-T) } \\
\text { questionnaire. }\end{array}$ & $\begin{array}{l}\text { Results showed moderate } \\
\text { convergent validity by } \\
\text { correlating fatigue with } \\
\text { quality of life, } \\
\text { including sleep. }\end{array}$ & $\begin{array}{l}\text { Results support the use of } \\
\text { the MFI traditional Chinese } \\
\text { version as an integral } \\
\text { instrument to measure } \\
\text { specific fatigue aspects. }\end{array}$ \\
\hline
\end{tabular}


Table 2. Cont.

\begin{tabular}{|c|c|c|c|c|c|c|}
\hline $\begin{array}{l}\text { Mental Load or } \\
\text { Fatigue Instrument }\end{array}$ & Authors & Sample & & $\begin{array}{l}\text { struments Used to } \\
\text { Compare }\end{array}$ & Results & Conclusions \\
\hline \multirow{6}{*}{ MFI } & Chung et al. (2014) & $\begin{array}{l}137 \text { major depressive } \\
\text { disorder }(\mathrm{MDD}) \\
\text { patients }(M=49.6 \\
S D=9.6)\end{array}$ & $\begin{array}{l}2 . \\
3 . \\
4 .\end{array}$ & $\begin{array}{l}\text { Scale to assess the } \\
\text { severity of Major } \\
\text { Depression and } \\
\text { Associated Symptoms } \\
\text { (HDRS) } \\
\text { HADS, } \\
\text { Insomnia Symptom } \\
\text { Self-Assessment Scale } \\
\text { (ISI) } \\
\text { SF-36. }\end{array}$ & $\begin{array}{l}\text { MFI-20 has good internal } \\
\text { consistency (Cronbach's } \\
\text { alpha }=0.89 \text { ). Suitable } \\
\text { concurrent validity, } \\
\text { significant correlations } \\
\text { between MFI-20 scores } \\
\text { and depressive and } \\
\text { anxiety symptoms, } \\
\text { general health, and quality } \\
\text { of life. }\end{array}$ & $\begin{array}{l}\text { MFI-20 is a valid and } \\
\text { reliable instrument to } \\
\text { assess fatigue in MDD } \\
\text { patients with residual } \\
\text { symptoms. }\end{array}$ \\
\hline & Elbers et al. (2012) & $\begin{array}{l}153 \text { patients } \\
\text { diagnosed with } \\
\text { Parkinson's disease } \\
(M=67.07 \\
S D=7.54)\end{array}$ & No & & $\begin{array}{l}\text { All subscales showed } \\
\text { suitable internal } \\
\text { consistency reflected by a } \\
\text { Cronbach range of } 0.74 \\
\text { to } 0.92 \text {. }\end{array}$ & $\begin{array}{l}\text { MFI is a reliable and valid } \\
\text { instrument to evaluate the } \\
\text { multidimensional aspects } \\
\text { of fatigue in Parkinson } \\
\text { patients. }\end{array}$ \\
\hline & $\begin{array}{l}\text { Hagelin et al. } \\
(2007)\end{array}$ & $\begin{array}{l}594 \text { cancer patients } \\
(M=59.50)\end{array}$ & 1. & Borg Scale (CR-10). & $\begin{array}{l}\text { Cronbach values in the } \\
\text { MFI- } 20 \text { ranged between } \\
0.67 \text { and } 0.94 \text {. The } \\
\text { correlation between the } \\
\text { MFI-20 subscales and the } \\
\text { CR- } 10 \text { scores ranged } \\
\text { between } 0.37 \text { and } 0.74 \text {. }\end{array}$ & $\begin{array}{l}\text { MFI-20 Swedish version is } \\
\text { a valid and reliable } \\
\text { instrument for measuring } \\
\text { fatigue in different patient } \\
\text { populations and in } \\
\text { healthy individuals. }\end{array}$ \\
\hline & Gentile et al. (2003) & $\begin{array}{l}225 \text { participants } \\
(M=52, S D=15)\end{array}$ & 1. & VAS & $\begin{array}{l}\text { Correlations between each } \\
\text { subscale and VAS are } \\
\text { highly significant } \\
(p<0.001)\end{array}$ & $\begin{array}{l}\text { MFI French version shows } \\
\text { that this instrument is valid } \\
\text { for clinical application. }\end{array}$ \\
\hline & Munch et al. (2006) & $\begin{array}{l}278 \text { advanced cancer } \\
\text { patients }(M=64)\end{array}$ & 1. & HADS. & $\begin{array}{l}\text { The two psychological } \\
\text { subscales of MFI-20, } \\
\text { Mental Fatigue and } \\
\text { Reduced Motivation, were } \\
\text { significantly associated } \\
\text { with each other. Only } \\
\text { General Fatigue and } \\
\text { Mental Fatigue correlated } \\
\text { significantly with the } \\
\text { HADS Anxiety subscale. }\end{array}$ & $\begin{array}{l}\text { MFI-20 may be a useful } \\
\text { tool for further research } \\
\text { on fatigue etiology. }\end{array}$ \\
\hline & $\begin{array}{l}\text { Schubart et al. } \\
\text { (2019) }\end{array}$ & $\begin{array}{l}175 \text { patients with } \\
\text { Ehlers-Danlos } \\
\text { Syndromes } \\
(M=42.40)\end{array}$ & $\begin{array}{l}1 . \\
2 . \\
3 . \\
4 . \\
5 . \\
6 .\end{array}$ & $\begin{array}{l}\text { Wisconsin Brief Pain } \\
\text { Inventory } \\
\text { Epworth Sleepiness } \\
\text { Scale (ESS) } \\
\text { PSQI } \\
\text { Beighton Score } \\
\text { Psychological } \\
\text { Inventory (SCL-90) } \\
\text { Sleep Medicine } \\
\text { Associates of } \\
\text { Maryland }\end{array}$ & $\begin{array}{l}\text { Mental fatigue was } \\
\text { correlated with pain } \\
(r=0.16) \text {, night sleep } \\
(r=0.20) \text {, daily sleep } \\
(r=0.35) \text {, and } \\
\text { dysautonomia }(r=0.36) .\end{array}$ & $\begin{array}{l}\text { This research shows the } \\
\text { relation between mental } \\
\text { fatigue and other } \\
\text { constructs. }\end{array}$ \\
\hline $\begin{array}{l}\text { Pittsburgh } \\
\text { Fatigability Scale } \\
\text { (PFS). }\end{array}$ & Burke et al. (2018) & $\begin{array}{l}35 \text { healthy old people } \\
(M=73.77, S D=5.9)\end{array}$ & $\begin{array}{l}1 . \\
2 . \\
3 . \\
4 . \\
5 . \\
6 .\end{array}$ & $\begin{array}{l}\text { MFIS } \\
\text { HADS } \\
\text { PSQI } \\
\text { ESS } \\
\text { Montreal Cognitive } \\
\text { Assessment (MOCA) } \\
\text { Operation Span Task } \\
\text { (OSPAN) }\end{array}$ & $\begin{array}{l}\text { PFS mental fatigue } \\
\text { subscores highly } \\
\text { correlated with the EES } \\
\text { scores }(\rho=0.63, p<0.001) \text {. } \\
\text { PFS mental fatigue scores } \\
\text { also correlated with the } \\
\text { MFIS cognitive score } \\
(\rho=0.36, p=<0.05) \text {. }\end{array}$ & $\begin{array}{l}\text { The lack of correlation } \\
\text { between task-based } \\
\text { fatigability measures and } \\
\text { the PFS Mental subscale } \\
\text { may indicate that mental } \\
\text { fatigue is difficult to } \\
\text { capture using questions } \\
\text { about fatigue based on } \\
\text { previous or imaginary } \\
\text { experiences. }\end{array}$ \\
\hline $\begin{array}{l}\text { Mental Load (ML) } \\
\text { and Mental Effort } \\
\text { (ME) Questionnaire } \\
\text { of Students in Biology } \\
\text { Education } \\
\text { (StuMMBE-Q). }\end{array}$ & Krell (2017) & $\begin{array}{l}602 \text { students ( } 9 \text { and } \\
10 \text { school grades; } \\
\text { from } 13 \text { to } 18 \text { years } \\
\text { old; } 52 \% \text { females). }\end{array}$ & No & & $\begin{array}{l}\text { Results suggest that } \\
\text { StuMMBE-Q classifies } \\
\text { students who report low, } \\
\text { medium, and high levels } \\
\text { of ML and ME. }\end{array}$ & $\begin{array}{l}\text { Findings suggest that the } \\
\text { questionnaire measures } \\
\text { two theoretically } \\
\text { established cognitive load } \\
\text { dimensions (mental load } \\
\text { and mental effort) well. }\end{array}$ \\
\hline $\begin{array}{l}\text { Rating Scale Mental } \\
\text { Effort (RSME) }\end{array}$ & Lin and Cai (2009) & Drivers. & $\begin{array}{l}1 . \\
2 .\end{array}$ & $\begin{array}{l}\text { Electrocardiogram } \\
\text { (ECG) } \\
\text { Continuous Mental } \\
\text { Workload Scale } \\
\text { (CBC-MWL). }\end{array}$ & $\begin{array}{l}\text { Correlation coefficient } \\
\text { between RSME and ECG } \\
\text { is } 0.85 \text {. ECG and } \\
\text { CBC-MWL measurement } \\
\text { show a high correlation } \\
\text { with the RSME score. }\end{array}$ & $\begin{array}{l}\text { Proposed method is } \\
\text { consistent with the RSME } \\
\text { method but RSME cannot } \\
\text { be completed in real time. }\end{array}$ \\
\hline
\end{tabular}


Table 2. Cont.

\begin{tabular}{|c|c|c|c|c|c|c|}
\hline $\begin{array}{c}\text { Mental Load or } \\
\text { Fatigue Instrument }\end{array}$ & Authors & Sample & & $\begin{array}{l}\text { struments Used to } \\
\text { Compare }\end{array}$ & Results & Conclusions \\
\hline $\begin{array}{l}\text { Situational Fatigue } \\
\text { Scale }\end{array}$ & $\begin{array}{l}\text { Yang and Wu } \\
\text { (2005) }\end{array}$ & $\begin{array}{l}96 \text { patients }(M=31.10, \\
S D=10.0) \text { and } 62 \\
\text { university students } \\
(M=21.0, S D=1.99)\end{array}$ & $\begin{array}{l}1 . \\
2 . \\
3 .\end{array}$ & $\begin{array}{l}\text { Fatigue Assessment } \\
\text { Instrument } \\
\text { Mental Fatigue } \\
\text { Subscale } \\
\text { Physical Fatigue } \\
\text { Subscale (PFSubscale) }\end{array}$ & $\begin{array}{l}\text { Cronbach coefficients } \\
\text { indicated good internal } \\
\text { consistency for the global } \\
\text { scale }(0.90) \text {, as well as for } \\
\text { the PFSubescale }(0.88) \text { and } \\
\text { the MFS }(0.89) \text {. }\end{array}$ & $\begin{array}{l}\text { SFS presents a new way to } \\
\text { measure fatigue } \\
\text { dimension that is different } \\
\text { from what is measured } \\
\text { with conventional fatigue } \\
\text { rating scales. }\end{array}$ \\
\hline $\begin{array}{l}\text { Somatic and } \\
\text { Psychological Health } \\
\text { Report. }\end{array}$ & $\begin{array}{l}\text { Couvy-Duchesne } \\
\text { et al. (2017) }\end{array}$ & $\begin{array}{l}5148 \text { participants } \\
(M=15.52 ; S D=0.75)\end{array}$ & No & & $\begin{array}{l}\text { Questionnaire could be } \\
\text { reduced to } 21 \text { items. }\end{array}$ & $\begin{array}{l}\text { This questionnaire could } \\
\text { be relevant to assess } \\
\text { anxiety, depression, and } \\
\text { chronic fatigue. }\end{array}$ \\
\hline $\begin{array}{l}\text { State-Trait Inventory } \\
\text { for Cognitive Fatigue } \\
\text { (STI-CF). }\end{array}$ & $\begin{array}{l}\text { Shuman-Paretsky } \\
\text { et al. (2017) }\end{array}$ & $\begin{array}{l}175 \text { participants, over } 65 \\
\text { years old }(M=77.35 \\
S D=6.91)\end{array}$ & $\begin{array}{l}1 . \\
2 . \\
3 . \\
\mathrm{RB}\end{array}$ & $\begin{array}{l}\text { Brief Fatigue } \\
\text { Inventory } \\
\text { Geriatric Depression } \\
\text { Scale } \\
\text { Trail Making Test } \\
\text { JS }\end{array}$ & $\begin{array}{l}\text { The } 4 \text { components } \\
\text { (cognitive fatigue, mental } \\
\text { effort, motivation, and } \\
\text { boredom) had good } \\
\text { reliability. Strong positive } \\
\text { relationship between } \\
\text { cognitive fatigue and a } \\
\text { subjective measure of } \\
\text { general fatigue }(p<0.001) \text {. }\end{array}$ & $\begin{array}{l}\text { The STI-CF had significant } \\
\text { relationships in the expected } \\
\text { direction with several } \\
\text { variables of cognitive and } \\
\text { health outcomes. }\end{array}$ \\
\hline $\begin{array}{l}\text { Subjective Exercise } \\
\text { Experiences Scale } \\
\text { (SEES). }\end{array}$ & $\begin{array}{l}\text { MeAuley and } \\
\text { Courneya (1994) }\end{array}$ & $\begin{array}{l}454 \text { university } \\
\text { students }(M=20.78 \\
S D=2.18)\end{array}$ & No & & $\begin{array}{l}\text { The comparison between } \\
\text { the three scales of SEES } \\
\text { showed their reliability: } \\
\text { Positive Well-Being (PWB) } \\
\alpha=0.36 \text {, Psychological } \\
\text { Distress (PD) } \alpha=0.25 \\
\text { and Fatigue } \alpha=0.88 \text {. }\end{array}$ & $\begin{array}{l}\text { Three dimensions of the } \\
\text { SEES provide initial support } \\
\text { for the multidimensional } \\
\text { measurement of the capacity } \\
\text { of psychological response to } \\
\text { the properties of exercise } \\
\text { stimulus: Positive } \\
\text { well-being, psychological } \\
\text { distress, and fatigue. }\end{array}$ \\
\hline $\begin{array}{l}\text { Subjective Scale of } \\
\text { Mental Workload } \\
\text { (SCA) }\end{array}$ & $\begin{array}{l}\text { Ceballos-Vásquez } \\
\text { et al. (2016) }\end{array}$ & $\begin{array}{l}379 \text { workers }(M=37.36 \\
S D=10.53) \text { of Critical } \\
\text { Patient Units (UPC) of } \\
\text { three Chilean hospitals. }\end{array}$ & 1. & $\begin{array}{l}\text { SUSESO-ISTAS } 21 \\
\text { questionnaire. }\end{array}$ & $\begin{array}{l}\text { There are positive and } \\
\text { significant correlations } \\
\text { between the global mental } \\
\text { load scores and all the } \\
\text { psychosocial dimensions } \\
\text { of the SUSESO-ISTAS } 21 \\
(p<0.05) \text {. }\end{array}$ & $\begin{array}{l}\text { SCAM presents high } \\
\text { reliability and suitable } \\
\text { validity in a Chilean sample } \\
\text { for mental load evaluation. }\end{array}$ \\
\hline
\end{tabular}

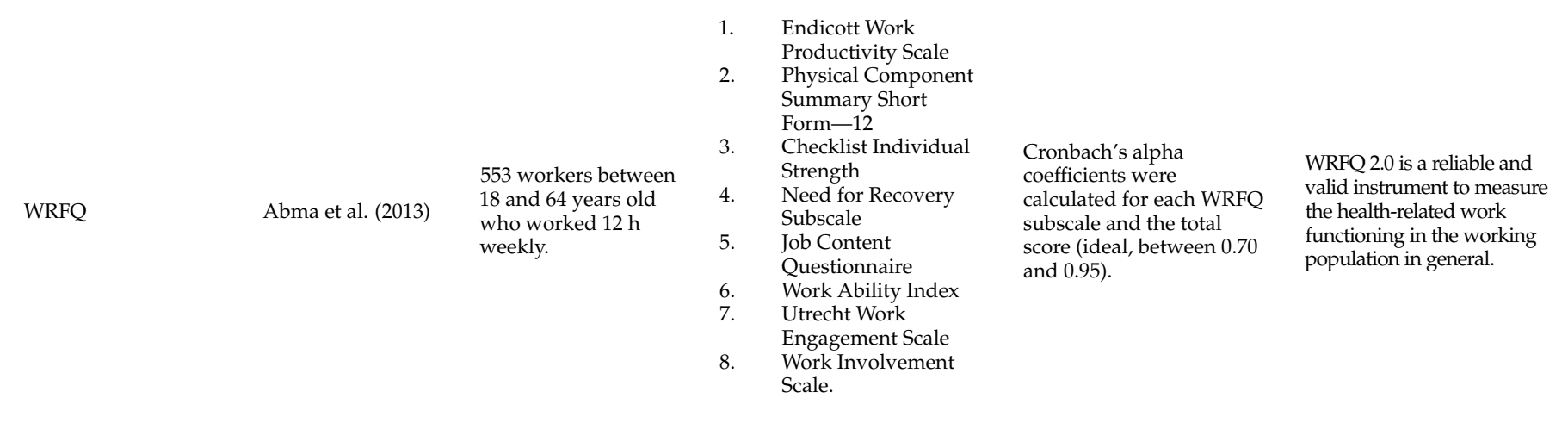

\subsection{Mental Load and Fatigue Assessment Instruments for Behavioral Derivations}

Table 3 presents five studies that used instruments to analyze the behavioral derivations of mental load and fatigue. Of these works, $12.5 \%$ focused on these derivations. Cognitive functioning, using attention, eye movement, accuracy, performance drive, or reaction time, was analyzed in this type of derivation of mental fatigue. To validate these instruments for the analysis of the behavioral derivations of mental fatigue, they were compared both with scales and questionnaires (e.g., MFS or CFS), other behavioral consequences (i.e., sleep), and physiological derivations (i.e., EEG). Most of these studies were performed with healthy participants, university students, or clinical patients, showing a higher variety of population than in the validation of the previously analyzed subjective scales. 
Table 3. Mental load and mental fatigue assessment instruments for behavioral derivations.

\begin{tabular}{|c|c|c|c|c|c|c|}
\hline $\begin{array}{l}\text { Mental Load or } \\
\text { Fatigue } \\
\text { Instrument }\end{array}$ & Authors & Sample & & $\begin{array}{l}\text { Instruments Used to } \\
\text { Compare }\end{array}$ & Results & Conclusions \\
\hline $\begin{array}{l}\text { Attentional } \\
\text { Function Index } \\
\text { (AFI) }\end{array}$ & $\begin{array}{l}\text { Cimprich et al. } \\
\text { (2011) }\end{array}$ & $\begin{array}{l}172 \text { women } \\
\text { diagnosed with } \\
\text { breast cancer. } \\
\text { Ages between } 27 \\
\text { and } 86 \text { years old. }\end{array}$ & No & & $\begin{array}{l}\text { Internal consistency } \\
\text { coefficient (Cronbach's } \\
\alpha) \text { for the revised } \\
\text { 13-item scale was } 0.92, \\
\text { indicating satisfactory } \\
\text { reliability. }\end{array}$ & $\begin{array}{l}\text { Findings indicate that AFI } \\
\text { is a valid and reliable } \\
\text { measure to assess the } \\
\text { perceived detrimental } \\
\text { effects of cognitive } \\
\text { dysfunction in chronic and } \\
\text { life-threatening diseases, } \\
\text { such as breast cancer. }\end{array}$ \\
\hline $\begin{array}{l}\text { Eyelink } 1000 \\
\text { Remote Eye } \\
\text { Tracking System }\end{array}$ & $\begin{array}{l}\text { Di Stasi et al. } \\
\text { (2012) }\end{array}$ & $\begin{array}{l}10 \text { healthy } \\
\text { volunteers. Five } \\
\text { women and five } \\
\text { men }(M=23.9 \\
S D=4.9)\end{array}$ & $\begin{array}{l}1 . \\
2 . \\
3 . \\
4 . \\
5 .\end{array}$ & $\begin{array}{l}\text { SIRCA Simulator } \\
\text { Groningen Sleep Qual- } \\
\text { ity Scale } \\
\text { Stanford Sleepiness Scale } \\
\text { CFS } \\
\text { Mental Workload Test }\end{array}$ & $\begin{array}{l}\text { The peak velocity of } \\
\text { saccadic eye } \\
\text { movements decreased } \\
\text { after driving }(p<0.05) \text {, } \\
\text { due to mental fatigue. }\end{array}$ & $\begin{array}{l}\text { Saccadic eye parameters, } \\
\text { particularly the peak } \\
\text { velocity, are a sensitive } \\
\text { indicator of mental fatigue. }\end{array}$ \\
\hline $\begin{array}{l}\text { Logitech Driving } \\
\text { Simulator with } \\
\text { Citycar Driving } \\
\text { software }\end{array}$ & $\begin{array}{l}\text { Puspasari et al. } \\
\text { (2017) }\end{array}$ & $\begin{array}{l}\text { Seven commercial } \\
\text { drivers, between } \\
25 \text { and } 35 \text { years } \\
\text { old. }\end{array}$ & $\begin{array}{l}1 . \\
2 .\end{array}$ & $\begin{array}{l}\text { Electroencephalogram } \\
\text { (EEG) } \\
\text { Karolinska Sleepiness } \\
\text { Scale }\end{array}$ & $\begin{array}{l}\text { All measured } \\
\text { parameters showed } \\
\text { significant changes } \\
\text { related to driving } \\
\text { duration }(p<0.05) .\end{array}$ & $\begin{array}{l}\text { Results show alpha, beta, } \\
\text { theta, and delta bands are } \\
\text { significantly different } \\
\text { before and after driving, } \\
\text { with an increase in the } \\
\text { theta-delta band and a } \\
\text { decrease in the alpha-beta } \\
\text { band. These correlate } \\
\text { with poor driving } \\
\text { performance. }\end{array}$ \\
\hline $\begin{array}{l}\text { Psychomotor } \\
\text { Vigilance Test } \\
\text { (PVT) }\end{array}$ & $\begin{array}{l}\text { Price et al. } \\
\text { (2017) }\end{array}$ & $\begin{array}{l}21 \text { participants } \\
(M=22, S D=4)\end{array}$ & $\begin{array}{l}1 . \\
2 . \\
3 .\end{array}$ & $\begin{array}{l}\text { Mental Arithmetic Test } \\
\text { Spatial Span Test } \\
\text { MFS }\end{array}$ & $\begin{array}{l}\text { Only the mobile test } \\
\text { PVT is valid and } \\
\text { reliable to assess } \\
\text { cognitive accuracy. The } \\
\text { arithmetic test does not } \\
\text { show a strong } \\
\text { correlation with MFS. }\end{array}$ & $\begin{array}{l}\text { The mobile application is } \\
\text { considered a potentially } \\
\text { effective tool for the } \\
\text { individual assessment of } \\
\text { cognitive fatigue levels } \\
\text { More continuity in time is } \\
\text { needed and the test must } \\
\text { be carried out daily. }\end{array}$ \\
\hline TRT_S software & $\begin{array}{l}\text { Crocetta et al. } \\
\text { (2014) }\end{array}$ & $\begin{array}{l}216 \text { university } \\
\text { students, between } \\
17 \text { and } 45 \text { years old } \\
(M=24, S D=6) .\end{array}$ & 1. & $\begin{array}{l}\text { Vienna } \\
\text { (VTS) }\end{array}$ & $\begin{array}{l}\text { Intraclass coefficient } \\
\text { correlation of TRT in } \\
\text { young adults showed a } \\
\text { strong correlation } \\
\text { between Simple TRT } \\
\text { and VTS }(r=0.72) .\end{array}$ & $\begin{array}{l}\text { Results confirmed the } \\
\text { TRT_S } 2012 \text { software's } \\
\text { validity, as a reliable } \\
\text { cognitive test to assess } \\
\text { the influence of mental } \\
\text { fatigue on cognitive } \\
\text { performance. }\end{array}$ \\
\hline
\end{tabular}

\subsection{Mental Load and Fatigue Assessment Instruments for Physiological Derivations}

Finally, Table 4 presents a total of five studies that designed a battery of tests or instruments to analyze the physiological derivations of mental fatigue. Of these studies, $12.5 \%$ focused on such derivations. The main instrument used for these derivations was the EEG. Concerning the comparison of instruments, some of these investigations used behavioral responses to compare the instruments, for example, a comparison of VAS and EEG. Samples of workers and healthy patients were used in these studies. 
Table 4. Mental load and mental fatigue assessment instruments for objective derivations.

\begin{tabular}{|c|c|c|c|c|c|c|}
\hline $\begin{array}{l}\text { Mental Load or } \\
\text { Fatigue Instrument }\end{array}$ & Authors & Sample & Inst & $\begin{array}{l}\text { uments Used to } \\
\text { Compare }\end{array}$ & Results & Conclusions \\
\hline $\begin{array}{l}\text { Air Traffic Workload } \\
\text { Input Technique }\end{array}$ & Neal et al. (2014) & $\begin{array}{l}16 \text { licensed air } \\
\text { traffic controllers. }\end{array}$ & 1. & $\begin{array}{l}\text { Task Load Met- } \\
\text { rics. }\end{array}$ & $\begin{array}{l}\text { The model explained } \\
42 \% \text { of the variance in } \\
\text { workload after } \\
\text { controlling for } \\
\text { differences among raters. }\end{array}$ & $\begin{array}{l}\text { The final model provided } \\
\text { a reasonable fit to the } \\
\text { data, despite including } \\
\text { only five predictors. It } \\
\text { can thus be considered a } \\
\text { multilevel unified } \\
\text { dynamic density model. }\end{array}$ \\
\hline $\begin{array}{l}\text { Cognitive } \\
\text { Pilot-Aircraft } \\
\text { Interface (CPAI) } \\
\text { procedures. }\end{array}$ & Liu et al. (2016) & Airplane pilots. & No & & $\begin{array}{l}\text { Higher heart rates are } \\
\text { related to higher fatigue } \\
\text { levels and the flickering } \\
\text { speed demonstrates a } \\
\text { similar relationship. For } \\
\text { mental fatigue, the heart } \\
\text { rate is more important } \\
\text { than the blink rate. }\end{array}$ & $\begin{array}{l}\text { Simulation results } \\
\text { demonstrate a } \\
\text { preliminary validity of } \\
\text { CPAI system for this } \\
\text { purpose. Estimated } \\
\text { human cognitive states } \\
\text { are consistent both with } \\
\text { external conditions and } \\
\text { physiological states. }\end{array}$ \\
\hline \multirow[t]{3}{*}{ EEG } & $\begin{array}{l}\text { Gharagozlou } \\
\text { et al. (2015) }\end{array}$ & $\begin{array}{l}12 \text { healthy male } \\
\text { drivers ( } M=23.8, \\
S D=1.44 ; \text { from } 20 \\
\text { to } 30 \text { years old). } \\
\text { Subjects had a } \\
\text { valid driver's } \\
\text { license with at least } \\
2 \text { years driving } \\
\text { experience and had } \\
\text { no brain injuries } \\
\text { history. }\end{array}$ & 1. & VAS. & $\begin{array}{l}\text { Significant increase in } \\
\text { absolute alpha power } \\
(p=0.006) \text {, as well as in } \\
\text { F-VAS scores were } \\
\text { observed during the } \\
\text { final driving section } \\
(p=0.001) \text {. }\end{array}$ & $\begin{array}{l}\text { The study suggested that } \\
\text { variations in alpha power } \\
\text { could be a good indicator of } \\
\text { drivers' mental fatigue. }\end{array}$ \\
\hline & Patel et al. (2018) & $\begin{array}{l}18 \text { participants of } \\
\text { different jobs }\end{array}$ & 1. & ECG. & $\begin{array}{l}\text { The use of EEG spectral } \\
\text { power in all bands } \\
\text { obtains better } \\
\text { performance for mental } \\
\text { fatigue assessment } \\
(p<0.001) \text {. }\end{array}$ & $\begin{array}{l}\text { The use of EEG spectral } \\
\text { power characteristics } \\
\text { across the entire range of } \\
\text { physiological bands } \\
\text { allows a better } \\
\text { representation of all } \\
\text { mental states. }\end{array}$ \\
\hline & Sun et al. (2014) & $\begin{array}{l}26 \text { right-handed and } \\
\text { neurologically } \\
\text { normal participants } \\
(M=22.20 \\
S D=1.53) .\end{array}$ & No & & $\begin{array}{l}\text { Few functional } \\
\text { connections were } \\
\text { significantly associated } \\
\text { with mental fatigue } \\
(p>0.05)\end{array}$ & $\begin{array}{l}\text { Viability demonstration } \\
\text { of a method of assessing } \\
\text { mental fatigue based on } \\
\text { functional connectivity. }\end{array}$ \\
\hline
\end{tabular}

\section{Discussion}

The aim of the present study was to summarize the different mental load and fatigue assessment instruments used, as well as to show their accuracy, reliability, and validity according to the derivation of mental load or fatigue analyzed by these instruments. The main results showed that there is a prevalence of subjective scales to measure mental load and fatigue. However, the use of electroencephalograms appears as an emergent form to understand the biological mechanisms of mental load and fatigue.

\subsection{Mental Load and Mental Fatigue Assessment Instruments for Subjective Derivations}

Our results showed that $75 \%$ of the instruments included in the present study focused on the subjective derivations of mental load and fatigue. These results indicated a tendency to use self-reported questionnaires or scales in the analysis of mental load and fatigue. The extended use of these types of instruments may be explained by the high validity and usefulness of their measurements [63]. However, experts should take into account the context involved to choose the most valid instrument, according to the data to be extracted. Previously, Russell et al. [8] defined the complex nature of human factors, which could explain why, when analyzing mental fatigue, experts also analyzed other psychological factors. Indeed, work settings and hospitals were the main contexts where these instruments have been used, whereas in other contexts, such as schools or sports, where 
mental fatigue is present $[64,65]$, few papers have analyzed the validity and reliability of these instruments [7]. As mentioned, these types of instruments are useful in the research of students and athletes because these populations usually have little time to answer our research questions [1]. The main interest of these instruments is the individualization of the feelings of mental fatigue [8]. Such individualization of the context is important from a clinical and practical viewpoint. For example, in a sports context, one task may significantly increase the mental fatigue of a certain athlete, while this same task will not change the mental fatigue of another athlete. This may be extended to hospital patients, students, or workers because mental fatigue has a subjective derivation, among others. Indeed, this situation justifies the use of these scales. However, although these types of instruments have highlighted the role of mental fatigue and promoted the study of this variable, a great number of experts have declared that further analysis of the physiological mechanisms is needed to explain mental load and fatigue [1].

\subsection{Mental Load and Fatigue Assessment Instruments for Behavioral Derivations}

Our results show that $12.5 \%$ of the instruments included in the present study focused on the behavioral derivations of mental load and fatigue. These variables allow experts to determine how mental fatigue may influence performance indicators in each context. Russell et al. [66] asked an athletic population about their symptoms in the presence of mental fatigue. These athletes felt slower, with poor reaction times and decreased accuracy. Moreover, a great number of papers have demonstrated the relationship between an increase in the feelings of mental fatigue and a decrease in the specific behavioral performance in different areas $[34,52,67]$. On the contrary, the results of the present study suggest that few instruments have been validated for this purpose from a behavioral perspective. From a clinical and practical point of view, this implies a limitation in the analysis of the negative effects of mental fatigue. Mental fatigue is important because of its negative consequences in surgeons, athletes, or performance and health drivers. More studies designing instruments for behavioral derivations or examining the effects of mental fatigue in human behavior are necessary to further analyze the importance of mental fatigue.

\subsection{Mental Load and Fatigue Assessment Instruments for Physiological Derivations}

Finally, our results show that $12.5 \%$ of the instruments included in the present study focused on the physiological derivations of mental load and fatigue. The influence of the brain in mental fatigue has been demonstrated; indeed, this influence has allowed researchers to differentiate the mental and physical nature of fatigue [2]. Whereas physical fatigue is normally caused by an impairment in the traditional physiological systems, such as heartrate or blood lactate, impairments in these systems have not been observed in the performance-related decreases in mental fatigue [2]. This shows that less is known about the psychobiological processes involved in mental fatigue. Although the complexity of these instruments (price, complexity, time ... ) could explain the few papers published about these instruments, this information would allow researchers to understand the mechanisms that underly the presence of mental fatigue and its consequences [7]. This information is interesting from a clinical and practical viewpoint. For example, it would be useful to know how mental fatigue can be manipulated, how recovery strategies can be used, or how to maintain performance in presence of mental fatigue. Indeed, as mentioned, a large number of experts support the importance of further analysis of this derivation to advance in this topic.

\section{Strengths and Limitations}

This investigation presents a series of noteworthy strengths. Firstly, to our knowledge, no previous studies have studied the instrument used to analyze mental load and fatigue. Indeed, no previous studies have classified the instruments used to analyze mental fatigue according to the type of derivation. 
The present research also presents some limitations that should be mentioned. The main limitation of this systematic review is the difficulty to obtain definitive conclusions, based on the heterogeneity of the type of instruments (e.g., scales, EEG, questionnaires).

\section{Practical Applications and Future Research}

The main practical application of this investigation is that these data could allow experts to choose an adequate instrument to analyze mental fatigue according to their needs. Experts could even design a battery of instruments to analyze mental fatigue from a global perspective.

For future research, we highlight the need to design specific instruments to quantify mental load and fatigue in sports or education. In addition, the use of behavioral and objective measures (e.g., blood sample or EEG) would allow further analysis of the causes and consequences of mental load and fatigue.

\section{Conclusions}

Of the studies included, $76 \%$ focused on the subjective derivation of mental fatigue. Therefore, we can conclude that most of the existing instruments to analyze mental load and fatigue are subjective questionnaires and scales. Furthermore, $12.5 \%$ analyzed the behavioral derivation, and $12.5 \%$ analyzed the physiological derivation of mental load and fatigue. Thus, few studies have designed instruments to quantify these variables from behavioral and physiological derivations. The scales have allowed experts to highlight the role of mental fatigue, which is important to assess the individual effect of mental load and fatigue in each subject. However, experts also stress the need to study the mechanisms involved in mental load and fatigue, analyzing the physiological mechanisms. More information is also necessary for sports and schools to analyze mental fatigue because most of these works were carried out with hospital patients and workers.

Author Contributions: Conceptualization, J.D.-G., I.G.-P., A.R.-M. and T.G.-C.; methodology, J.C.P.-B., M.Á.L.-G. and I.R.-B.; formal analysis, J.D.-G., J.C.P.-B., M.Á.L.-G. and I.R.-B.; investigation, I.G.-P.; resources, T.G.-C.; data curation, J.C.P.-B., M.Á.L.-G. and I.R.-B.; writing-original draft preparation, J.D.-G.; writing-review and editing, J.C.P.-B., M.Á.L.-G. and I.R.-B.; visualization, A.R.-M.; supervision, I.G.-P. and T.G.-C. All authors have read and agreed to the published version of the manuscript.

Funding: This work was supported by the Assistance to Research Groups (GR18102) of the Junta de Extremadura (Ministry of Employment and Infrastructure); with the contribution of the European Union through the European Regional Development Funds (ERDF). This research was also supported by an FPU PhD candidate grant from the Government of Spain (Ministry of Education, Culture, and Sports) to Díaz, J. (FPU18/03660).

Institutional Review Board Statement: Not applicable.

Informed Consent Statement: Not applicable.

Data Availability Statement: Not applicable.

Conflicts of Interest: The authors declare no conflict of interest.

\section{References}

1. Ishii, A.; Tanaka, M.; Watanabe, Y. Neural mechanisms of mental fatigue. Rev. Neurosci. 2014, 25, 469-479. [CrossRef]

2. Van Cutsem, J.; Roelands, B.; Pluym, B.; Tassignon, B.; Verschueren, J.O.; De Pauw, K.; Meeusen, R. Can creatine combat the mental fatigue-associated decrease in visuomotor skills? Med. Sci. Sports Exerc. 2020, 52, 120-130. [CrossRef]

3. Pageaux, B.; Lepers, R. The effects of mental fatigue on sport-related performance. Prog. Brain Res. 2018, 240, 291-315. [PubMed]

4. Hopstaken, J.F.; van der Linden, D.; Bakker, A.B.; Kompier, M.A. A multifaceted investigation of the link between mental fatigue and task disengagement. Psychophysiology 2015, 52, 305-315. [CrossRef] [PubMed]

5. Tran, Y.; Craig, A.; Craig, R.; Chai, R.; Nguyen, H. The influence of mental fatigue on brain activity: Evidence from a systematic review with meta-analyses. Psychophysiology 2020, 57, e13554. [CrossRef] [PubMed]

6. Van Cutsem, J.; Marcora, S.; De Pauw, K.; Bailey, S.; Meeusen, R.; Roelands, B. The effects of mental fatigue on physical performance: A systematic review. Sport Med. 2017, 47, 1569-1588. [CrossRef] 
7. Van Cutsem, J.; Marcora, S. The effects of mental fatigue on sport performance. In Motivation and Self-Regulation in Sport and Exercise; Routledge: London, UK, 2021; pp. 134-148.

8. Russell, S.; Kelly, V.G.; Halson, S.L.; Jenkins, D.G. Cognitive load in sport. Human Factors and Ergonomics in Sport: Applications and Future Directions; CRC Press: London, UK, 2020; pp. 181-200.

9. McMorris, T. Cognitive fatigue effects on physical performance: The role of interoception. Sport Med. 2020, 50, 1703-1708. [CrossRef] [PubMed]

10. Li, J.; Li, H.; Wang, H.; Umer, W.; Fu, H.; Xing, X. Evaluating the impact of mental fatigue on construction equipment operators' ability to detect hazards using wearable eye-tracking technology. Autom. Constr. 2019, 105, 102835. [CrossRef]

11. Ceballos-Vásquez, P.; Rolo-González, G.; Hernández-Fernaud, E.; Díaz-Cabrera, D.; Paravic-Klijn, T.; Burgos-Moreno, M.; Barriga, O. Validation of the Subjective Scale of Mental Workload (ESCAM) in health professionals in Chile. Univ. Psychol. 2016, 15, $261-270$.

12. Krell, M. Evaluating an instrument to measure mental load and mental effort considering different sources of validity evidence Cogent Educ. 2017, 4, 1280256. [CrossRef]

13. Sampei, K.; Ogawa, M.; Torres, C.; Sato, M.; Miki, N. Mental fatigue monitoring using a wearable transparent eye detection system. Micromachines 2016, 7, 20. [CrossRef]

14. Käthner, I.; Wriessnegger, S.C.; Müller-Putz, G.R.; Kübler, A.; Halder, S. Effects of mental workload and fatigue on the P300, alpha and theta band power during operation of an ERP (P300) brain-computer interface. Biol. Psychol. 2014, 102, 118-129. [CrossRef] [PubMed]

15. Sun, Y.; Lim, J.; Meng, J.; Kwok, K.; Thakor, N.; Bezerianos, A. Discriminative analysis of brain functional connectivity patterns for mental fatigue classification. Ann. Biomed. Eng. 2014, 42, 2084-2094. [PubMed]

16. Grant, M.J.; Booth, A. A typology of reviews: An analysis of 14 review types and associated method-ologies. Health Inf. Libr. J. 2009, 26, 91-108. [CrossRef] [PubMed]

17. Liberati, A.; Altman, D.G.; Tetzlaff, J.; Mulrow, C.; Gøtzsche, P.C.; Ioannidis, J.P.A.; Clarke, M.; Devereaux, P.J.; Kleijnen, J.; Moher, D. The PRISMA statement for reporting systematic reviews and meta-analyses of studies that evaluate health care interventions: Explanation and elaboration. J. Clin. Epidemiol. 2009, 62, e1-e34. [CrossRef]

18. Moher, D.; Liberati, A.; Tetzlaff, J.; Altman, D.G. Preferred reporting items for systematic reviews and meta-analyses: The PRISMA statement. BMJ 2009, 339, 332-336. [CrossRef]

19. Booth, A. The pros and pros of registration on PROSPERO. BJOG Int. J. Obstet. Gynaecol. 2012, 119, $904-905$.

20. Booth, A.; Clarke, M.; Dooley, G.; Ghersi, D.; Moher, D.; Petticrew, M.; Stewart, L. PROSPERO at one year: An evaluation of its utility. Syst. Rev. 2013, 2, 4-11. [CrossRef]

21. Gough, D.; Oliver, S.; Thomas, J. An Introduction to Systematic Reviews, 2nd ed.; SAGE: London, UK, 2012.

22. Chalmers, I.; Altman, D.G. Systematic Reviews, 1st ed.; BMJ Publications: London, UK, 1995.

23. Cohen, J. Weighted kappa: Nominal scale agreement with provision for scaled disagreement or partial credit. Psychol. Bull. 1968, 70, 213-220. [CrossRef]

24. McHugh, M.L. Interrater reliability: The kappa statistic. Biochem. Med. 2012, 22, 276-282. [CrossRef]

25. Patel, A.N.; Howard, M.D.; Roach, S.M.; Jones, A.P.; Bryant, N.B.; Robinson, C.S.H.; Clark, V.P.; Pilly, P.K. Mental state assessment and validation using personalized physiological biometrics. Front. Hum. Neurosci. 2018, 12, 221. [PubMed]

26. Kmet, L.M.; Lee, R.C. Standard Quality Assessment Criteria for Evaluating Primary Research Papers from a Variety of Fields; Edmont Alberta Herritage Foundation for Medical Research: Edmonton, AB, Canada, 2004

27. Chilcot, J.; Norton, S.; Kelly, M.E.; Moss-Morris, R. The Chalder Fatigue Questionnaire is a valid and reliable measure of perceived fatigue severity in multiple sclerosis. Mult. Scler. J. 2016, 22, 677-684. [CrossRef] [PubMed]

28. Cho, H.J.; Costa, E.; Menezes, P.R.; Chalder, T.; Bhugra, D.; Wessely, S. Cross-cultural validation of the Chalder Fatigue Questionnaire in Brazilian primary care. J. Psychosom. Res. 2007, 62, 301-304. [PubMed]

29. Chiu, H.Y.; Li, W.; Lin, J.H.; Su, Y.K.; Lin, E.Y.; Tsai, P.S. Measurement properties of the Chinese version of the Mental Fatigue Scale for patients with traumatic brain injury. Brain Inj. 2018, 32, 652-664. [PubMed]

30. Duan, W.; Mu, W. Validation of a Chinese version of the stress overload scale-short and its use as a screening tool for mental health status. Qual. Life Res. 2018, 27, 411-421. [PubMed]

31. Fong, T.C.; Chan, J.S.; Chan, C.L.; Ho, R.T.; Ziea, E.T.; Wong, V.C.; Ng, B.F.; Ng, S.M. Psychometric properties of the Chalder Fatigue Scale revisited: An exploratory structural equation modeling approach. Qual. Life Res. 2015, 24, 2273-2278. [PubMed]

32. Visser-Keizer, A.C.; Hogenkamp, A.; Westerhof-Evers, H.J.; Egberink, I.J.; Spikman, J.M. Dutch multifactor fatigue scale: A new scale to measure the different aspects of fatigue after acquired brain injury. Arch. Phys. Med. Rehabil. 2015, 96, 1056-1063. [CrossRef]

33. Friedrich, M.; Nowe, E.; Hofmeister, D.; Kuhnt, S.; Leuteritz, K.; Sender, A.; Stobel-Richer, Y.; Geue, K. Psychometric properties of the fatigue questionnaire EORTC QLQ-FA12 and proposal of a cut-off value for young adults with cancer. Health Qual. Life Outcomes 2018, 16, 125. [CrossRef]

34. Knobel, H.; Loge, J.H.; Brenne, E.; Fayers, P.; Hjermstad, M.J.; Kaasa, S. The validity of EORTC QLQ-C30 fatigue scale in advanced cancer patients and cancer survivors. Palliat. Med. 2003, 17, 664-672. [CrossRef] [PubMed]

35. Porro, B.; Michel, A.; Zinzindohoué, C.; Bertrand, P.; Monrigal, E.; Trentini, F.; Baussard, L.; Cousson-Gélie, F. Quality of life, fatigue and changes therein as predictors of return to work during breast cancer treatment. Scand. J. Caring Sci. 2019, 33, 467-477. [PubMed] 
36. Zhang, M.; Sparer, E.H.; Murphy, L.A.; Dennerlein, J.T.; Fang, D.; Katz, J.N.; Caban-Martinez, A.J. Development and validation of a Fatigue Assessment Scale for U.S. construction workers. Am. J. Ind. Med. 2015, 58, 220-228. [CrossRef]

37. Kauffman, B.Y.; Garey, L.; Nordan, A.; Jardin, C.; Mayorga, N.A.; Robles, Z.; Zvolensky, M.J. The development and initial validation of the Fatigue Sensitivity Questionnaire. Cogn. Behav. Ther. 2019, 48, 419-429. [CrossRef]

38. Kumamoto, K.; Arai, Y. Validation of "personal strain" and "role strain": Subscales of the short version of the Japanese version of the Zarit Burden Interview (J-ZBI_8). Psychiatry Clin. Neurosci. 2004, 58, 606-610. [CrossRef]

39. Dębska, G.; Wilczek-Ruzyczka, E.; Foryś, Z.; Małgorzata, P. Psychometric properties assessment of the Meister questionnaire (Polish version) used in evaluating mental load among nurses. Med. Pracy 2013, 64, 349-358. [CrossRef]

40. Bertram, D.A.; Hersey, C.O.; Opila, D.A.; Quirin, O. A measure of physician mental work load in internal medicine ambulatory care clinics. Med. Care 1990, 28, 458-467. [CrossRef]

41. Chuang, L.L.; Chuang, Y.F.; Hsu, M.J.; Huang, Y.Z.; Wong, A.M.; Chang, Y.J. Validity and reliability of the traditional Chinese version of the multidimensional fatigue inventory in general population. PLoS ONE 2018, 13, e0189850. [CrossRef]

42. Chung, K.F.; Yu, B.Y.M.; Yung, K.P.; Yeung, W.F.; Ng, T.H.; Ho, F.Y.Y. Assessment of fatigue using the Multidimensional Fatigue Inventory in patients with major depressive disorder. Compr. Psychiatry 2014, 55, 1671-1678. [CrossRef] [PubMed]

43. Elbers, R.G.; van Wegen, E.E.H.; Verhoef, J.; Kwakkel, G. Reliability and structural validity of the Multidimensional Fatigue Inventory (MFI) in patients with idiopathic Parkinson's disease. Park. Relat. Disord. 2012, 18, 532-536. [CrossRef] [PubMed]

44. Hagelin, C.; Wengström, Y.; Runesdotter, S.; Fürst, C. The psychometric properties of the Swedish Multidimensional Fatigue Inventory MFI-20 in four different populations. Acta Oncol. 2007, 46, 97-104. [PubMed]

45. Gentile, S.; Delaroziere, J.C.; Favre, R.; Sambuc, R.; San Marco, J.L. Validation of the French "multidimensional fatigue inventory" (MFI 20). Eur. J. Cancer Care 2003, 12, 58-64. [CrossRef]

46. Munch, T.N.; Strömgren, A.S.; Pedersen, L.; Petersen, M.A.; Hoermann, L.; Groenvold, M. Multidimensional measurement of fatigue in advanced cancer patients in palliative care: An application of the Multidimensional Fatigue Inventory. J. Pain Symptom Manag. 2006, 31, 533-541. [CrossRef] [PubMed]

47. Schubart, J.R.; Schaefer, E.; Hakim, A.J.; Francomano, C.A.; Bascom, R. Use of cluster analysis to delineate symptom profiles in an Ehlers-Danlos Syndrome patient population. J. Pain Symptom Manag. 2019, 58, 427-436. [CrossRef] [PubMed]

48. Burke, S.E.; Samuel, I.B.H.; Zhao, Q.; Cagle, J.; Cohen, R.A.; Kluger, B.; Ding, M. Task-based cognitive fatigability for older adults and validation of mental fatigability subscore of pittsburgh fatigability scale. Front. Aging Neurosci. 2018, 10, 327. [PubMed]

49. Lin, Y.; Cai, H. A method for building a real-time cluster-based continuous mental workload scale. Issues Erg. Sci. 2009, 10, 531-543.

50. Yang, C.M.; Wu, C.H. The Situational Fatigue Scale: A different approach to measuring fatigue. Qual. Life Res. 2005, 14, 1357-1362.

51. Couvy-Duchesne, B.; Davenport, T.A.; Martin, N.G.; Wright, M.J.; Hickie, I.B. Validation and psychometric properties of the Somatic and Psychological HEalth REport (SPHERE) in a young Australian-based population sample using non-parametric item response theory. BMC Psychiatry 2017, 17, 279. [CrossRef]

52. Shuman-Paretsky, M.; Zemon, V.; Foley, F.W.; Holtzer, R. Development and validation of the State-Trait Inventory of Cognitive Fatigue in community-dwelling older adults. Arch. Phys. Med. Rehabil. 2017, 98, 766-773. [CrossRef]

53. MeAuley, E.; Courneya, K.S. The Subjective Exercise Experiences Scale (SEES): Development and preliminary validation. J. Sport Exerc. Psychol. 1994, 16, 163-177.

54. Abma, F.I.; Van Der Klink, J.J.; Bültmann, U. The Work Role Functioning Questionnaire 2.0 (Dutch Version): Examination of its reliability, validity and responsiveness in the general working population. J. Occup. Rehabil. 2013, 23, $135-147$.

55. Cimprich, B.; Visovatti, M.; Ronis, D.L. The attentional function index-a self-report cognitive measure. Psycho-Oncology 2011, 20, 194-202 [CrossRef]

56. Di Stasi, L.L.; Renner, R.; Catena, A.; Cañas, J.J.; Velichkovsky, B.M.; Pannasch, S. Towards a driver fatigue test based on the saccadic main sequence: A partial validation by subjective report data. Transp. Res. Part C Emerg. Technol. 2012, 21, 122-133. [CrossRef]

57. Puspasari, M.A.; Iridiastadi, H.; Sutalaksana, I.Z.; Sjafruddin, A. Effect of driving duration on EEG fluctuations. Int. J. Technol. 2017, 8, 1089-1096. [CrossRef]

58. Price, E.; Moore, G.; Galway, L.; Linden, M. Validation of a smartphone-based approach to in situ cognitive fatigue assessment. JMIR mHealth uHealth 2017, 5, e125.

59. Crocetta, T.B.; Viana, R.L.; Silva, D.E.; Monteiro, C.B.D.M.; Arab, C.; Andrade, A. Validade de software para medição do tempo de reação total com estímulo simples-TRT_S. J. Hum. Growth Dev. 2014, 24, 295-303.

60. Neal, A.; Hannah, S.; Sanderson, P.; Bolland, S.; Mooij, M.; Murphy, S. Development and validation of a multilevel model for predicting workload under routine and nonroutine conditions in an air traffic management center. Hum. Factors 2014, 56, 287-305.

61. Liu, J.; Gardi, A.; Ramasamy, S.; Lim, Y.; Sabatini, R. Cognitive pilot-aircraft interface for single-pilot operations. Knowledge-Based Syst. 2016, 112, 37-53.

62. Gharagozlou, F.; Saraji, G.N.; Mazloumi, A.; Nahvi, A.; Nasrabadi, A.M.; Foroushani, A.R.; Kheradmand, A.; Ashouri, M.; Samavati, M. Detecting driver mental fatigue based on EEG Alpha power changes during simulated driving. Iran. J. Public Health 2015, 44, 1693-1700.

63. Barte, J.C.M.; Nieuwenhuys, A.; Geurts, S.A.E.; Kompier, M.A.J. Motivation counteracts fatigue-induced performance decrements in soccer passing performance. J. Sports Sci. 2019, 37, 1189-1196. [CrossRef] [PubMed] 
64. Key, A.P.; Gustafson, S.J.; Rentmeester, L.; Hornsby, B.W.Y.; Bess, F.H. Speech-processing fatigue in children: Auditory eventrelated potential and behavioral measures. J. Speech Lang. Hear. Res. 2017, 60, 2090-2104. [CrossRef] [PubMed]

65. Thompson, C.J.; Noon, M.; Towlson, C.; Perry, J.; Coutts, A.J.; Harper, L.D.; Skorski, S.; Smith, M.R.; Barrett, S.; Meyer, T. Understanding the presence of mental fatigue in English academy soccer players. J. Sports Sci. 2020, 38, 1524-1530. [CrossRef] [PubMed]

66. Russell, S.; Jenkins, D.; Rynne, S.; Halson, S.L.; Kelly, V. What is mental fatigue in elite sport? Perceptions from athletes and staff. Eur. J. Sport Sci. 2019, 19, 1367-1376. [CrossRef] [PubMed]

67. Van Cutsem, J.; De Pauw, K.; Buyse, L.; Marcora, S.; Meeusen, R.; Roelands, B. Effects of mental fatigue on endurance performance in the heat. Med. Sci. Sports Exerc. 2017, 49, 294-306. [CrossRef] [PubMed] 